QUARTERLY OF APPLIED MATHEMATICS

VOLUME LXIII, NUMBER 4

DECEMBER 2005, PAGES 691-714

S 0033-569X(05)00977-9

Article electronically published on August 17, 2005

\title{
ANALYSIS OF THE RELATIVISTIC VLASOV-MAXWELL MODEL IN AN INTERVAL
}

\author{
BY \\ FRANCIS FILBET (Mathématiques pour l'Industrie et la Physique, Université Paul Sabatier, 118 \\ route de Narbonne, 31062, Toulouse cedex 04, France), \\ YAN GUO (Division of Applied Mathematics, Brown University, Providence, Rhode Island 02912), \\ AND \\ CHI-WANG SHU (Division of Applied Mathematics, Brown University, Providence, Rhode Island \\ 02912)
}

Abstract. The dynamics of a collisionless electron gas under the influence of the selfconsistent electromagnetic field is studied in an interval, where electrons are emitted at one end of the interval and absorbed at the other. The electron distribution $f$ depends on one space variable $x$ and two dimensional velocity variables $\left(v_{1}, v_{2}\right)$, whereas the electromagnetic field satisfies the Maxwell system. It is shown that the electromagnetic field is smooth enough to define the trajectories of particles. On the other hand, due to the absorbing boundary condition, $f$ is in general not smooth, and only has bounded total variations. Such $B V$ regularity is sufficient to guarantee the uniqueness of the nonlinear electron dynamics. Finally, numerical simulations are performed to demonstrate the formation of a singularity from the boundary for the electron distribution.

1. Introduction. In many problems encountered in plasma physics or beam propagation, the numerical resolution of the full Vlasov-Maxwell system can be extremely expensive in computer time. It is sometimes possible to use simplified models which approximate the Maxwell equations in some sense. Such situations are encountered, for instance, when no high frequency phenomenon occurs. A first approximation in this case would be the Poisson equation, which neglects altogether magnetic effects. However, the construction of particle accelerators and free electron lasers requires electron guns which

Received February 20, 2005.

2000 Mathematics Subject Classification. Primary 35Q72, 76X05, 82D99.

Key words and phrases. Vlasov-Maxwell system, boundary problem, BV estimate.

The research of Y. Guo was supported in part by NSF grant DMS-0305161 and by a Salomon award from Brown University. The research of C.-W. Shu was supported in part by ARO grant W911NF-04-1-0291, NSF grant DMS-0207451 and AFOSR grant F49620-02-1-0113.

E-mail address: filbet@mip.ups-tlse.fr

E-mail address: guoy@dam.brown.edu

E-mail address: shu@dam.brown.edu

(C)2005 Brown University 
produce relativistic electron beams of high quality (low emission and high current), and relativistic and magnetic effects cannot be neglected. In this case, we can use some symmetries of the geometry domain to reduce the dimension without neglecting any physical effects. It is the purpose of this article to investigate the dynamics of such an electron gas under the self-consistent magnetic effect.

The boundary in such a problem is always characteristic, so the question of uniqueness and regularity of the solution in the presence of a boundary is very challenging mathematically. In this paper, we study the one and one-half dimensional Vlasov-Maxwell system over an interval, which is the evolution model for classical electromagnetic conduction in a plane diode. Even in this simplest case, the regularity of such dynamics has been open.

Let a dilute electron gas be emitted at $x=0$ and absorbed at $x=1$. Under an external voltage, the dynamics of such a plane diode is modeled by the Vlasov-Maxwell system 13

$$
\frac{\partial f}{\partial t}+\hat{v}_{1}(v) \frac{\partial f}{\partial x}+(\mathbf{E}+\hat{v}(v) \times \mathbf{B}) \cdot \nabla_{v} f=0, \quad t \in \mathbb{R}^{+}, x \in(0,1), v \in \mathbb{R}^{2},
$$

with the relativistic velocity (with the speed of light normalized to be one)

$$
\hat{v}(v)=\frac{v}{\sqrt{1+v^{2}}}
$$

for $v=\left(v_{1}, v_{2}\right)$, and the distribution function $f\left(t, x, v_{1}, v_{2}\right)$ and electromagnetic field

$$
\mathbf{E}=\left(E_{1}, E_{2}, 0\right) \quad \mathbf{B}=(0,0, B)
$$

also satisfy the one-dimensional Maxwell system as

$$
\begin{aligned}
\frac{\partial E_{1}}{\partial t} & =-j_{1}(t, x) \equiv-\int_{\mathbb{R}^{2}} \hat{v}_{1}(v) f(t, x, v) d v \\
\frac{\partial E_{2}}{\partial t}+\frac{\partial B}{\partial x} & =-j_{2}(t, x) \equiv-\int_{\mathbb{R}^{2}} \hat{v}_{2}(v) f(t, x, v) d v \\
\frac{\partial B}{\partial t}+\frac{\partial E_{2}}{\partial x} & =0
\end{aligned}
$$

with the constraint

$$
\partial_{x} E_{1}=\rho(t, x) \equiv \int_{\mathbb{R}^{2}} f(t, x, v) d v .
$$

The macroscopic charged density and current density are denoted by $\rho$ and $j$ respectively.

The initial datum is

$$
f(0, x, v)=f_{0}(x, v) .
$$

Here the boundary conditions for the electron distribution are given by

$$
\begin{aligned}
& f(t, 0, v)=g(t, v), \quad v_{1}>0, \\
& f(t, 1, v)=0, \quad v_{1}<0,
\end{aligned}
$$

initial data are given for the electromagnetic fields

$$
E_{1}(0, x)=E_{1,0}(x), \quad E_{2}(0, x)=E_{2,0}(x), \quad B(0, x)=B_{0}(x), \quad x \in(0,1),
$$

and perfect conductor boundary conditions $(\mathbf{E} \times n=0, \mathbf{B} \cdot n=0)$ are prescribed for $E_{2}$,

$$
E_{2}(t, 0)=E_{2}(t, 1)=0, \quad t \in \mathbb{R}^{+} .
$$


Before describing our results, let us briefly mention that the Vlasov-Poisson and Vlasov-Maxwell equations have been studied by several authors: the existence of classical solutions has been obtained in [3, 17, 19, (Vlasov-Poisson) and 2, 8, 9, 10, (VlasovMaxwell). The existence of weak solutions is described in [1, 14, 16] (Vlasov-Poisson) and 5] (Vlasov-Maxwell). The mathematical study of nonlinear boundary value problems was initiated in the pioneering work in 13, in which stationary (independent of time t) solutions are constructed. Such stationary solutions are generically not continuous. A higher-dimensional generalization was given in [4] and [18].

Recently, Guo, Shu and Zhou [12] have given an analysis of the simple one-dimensional Vlasov-Poisson system with boundary condition and have shown that when the applied potential is strong enough the solution is only $B V$ and cannot be continuous. In the present work we extend this study to the one-dimensional Vlasov-Maxwell problem with boundary conditions, where the effect of the magnetic field is taken into account.

We now introduce some notation. Letting $T$ be an arbitrary positive constant, we define the region

$$
\Omega=(0,1) \times \mathbb{R}^{2}
$$

and

$$
\Pi=[0, T] \times[0,1] \times \mathbb{R}^{2}, \quad \Pi_{s}=\Pi \cap\{t=s\}, \quad 0 \leq s \leq T,
$$

and the incoming sets at the boundaries $\{x=0\}$ and $\{x=1\}$ as

$$
\gamma_{0}^{+}=\left\{(t, 0, v), \quad v_{1}(v)>0,0 \leq t \leq T\right\}, \quad \gamma_{1}^{-}=\left\{(t, 1, v), \quad v_{1}(v)<0,0 \leq t \leq T\right\} .
$$

We denote the singular set at the boundary as

$$
\gamma_{S}=\left\{x=0, v_{1}=0\right\} \cup\left\{x=1, v_{1}=0\right\} .
$$

We use $\|\cdot\|_{p}$ to denote the standard $L^{p}$ norms for $1 \leq p \leq \infty$, and let $C^{0,1}=W^{1, \infty}$ be the space of Lipschitz continuous functions. Let $D=\left[\partial_{t}, \partial_{x}, \partial_{v}\right]$.

Our main result for the nonlinear problem is as follows.

Theorem 1.1. Consider the nonlinear Vlasov-Maxwell system with boundary conditions (1.1)-(1.8). Assume $f_{0}(0, x, v) \in L^{\infty} \cap B V\left(\Pi_{0}\right), g(t, v) \in L^{\infty} \cap B V\left(\gamma_{0}^{+}\right)$are both compactly supported, and $\left(E_{1,0}, E_{2,0}, B_{0}\right) \in C^{0,1}([0,1])$. Then, there exists a unique weak solution $f \in B V \cap L^{\infty}$ and $E_{1}, E_{2}, B \in C^{0,1}$.

We remark that if the following compatibility conditions are valid

$$
f_{0}\left(0, v_{1}, v_{2}\right)=g\left(0, v_{1}, v_{2}\right), \quad v_{1}>0, \quad f_{0}\left(1, v_{1}, v_{2}\right)=0, \quad v_{1}<0,
$$

then no singularity would emanate from $t=0$ and $x=0$.

To prove this result, we first give a priori estimates on the electromagnetic fields $(E, B)$ and on the distribution function $f$. The key estimate is to obtain an $L^{\infty}$ bound on the fields that only depends on the total energy. We follow closely the idea of Glassey and Schaeffer for the whole line problem [8]. Due to the presence of the boundary condition, we have to separate the domain into four different regions and carefully estimate each region. Then, a $C^{0,1}$ bound on the electromagnetic fields allows us to define correctly the characteristic curves and to obtain uniform estimates on the trajectories. We next give a regularization of the linear Vlasov equation by adding a damping source term at the 
singularity point $(x, v)=(0,0)$ to get a uniform $B V$ bound on the distribution function. Then, using an iteration scheme and passing to the limit, we prove the existence of a weak solution to the Vlasov-Maxwell system, while the uniqueness of such a solution follows from the $B V$ property of the distribution function $f$. Finally, numerical results illustrate the formation of singularities coming from the boundary.

\section{Field estimates.}

2.1. $L^{\infty}$ estimate. Before giving an estimate on the electromagnetic fields we need to establish a global energy estimate.

Lemma 2.1. Consider $(f, E, B)$ a smooth solution of the Vlasov-Maxwell system (1.1)(1.4) with boundary conditions (1.5)-(1.8) on the time interval $[0, T]$. Then, the solution satisfies the energy estimate

$$
\begin{aligned}
& \int_{0}^{1} \int_{\mathbb{R}^{2}} \sqrt{1+|v|^{2}} f(t, x, v) d x d v+\frac{1}{2} \int_{0}^{1}|\mathbf{E}(t, x)|^{2}+|B(t, x)|^{2} d x \\
& -\int_{0}^{t} \int_{\left\{v_{1}<0\right\}} v_{1} f(\tau, 0, v) d v d \tau+\int_{0}^{t} \int_{\left\{v_{1}>0\right\}} v_{x} f(\tau, 1, v) d v d \tau \\
\leq & \int_{0}^{1} \int_{\mathbb{R}^{2}} \sqrt{1+|v|^{2}} f_{0} d x d v+\frac{1}{2} \int_{0}^{1}\left|\mathbf{E}_{0}(x)\right|^{2}+\left|B_{0}(x)\right|^{2} d x \\
& +\int_{0}^{t} \int_{\left\{v_{1}<0\right\}} v_{1} g(\tau, v) d v d \tau .
\end{aligned}
$$

Proof. We first get the energy identity by multiplying the Vlasov equation (1.1) by $\sqrt{1+|v|^{2}}$ and using Maxwell's equations (1.2)-(1.4)

$$
\frac{\partial e}{\partial t}-\frac{\partial m}{\partial x}=0,
$$

with the energy and momenta density given by

$$
\begin{aligned}
e & =\frac{1}{2}\left(|\mathbf{E}|^{2}+|B|^{2}\right)+\int_{\mathbb{R}^{2}} \sqrt{1+|v|^{2}} f d v, \\
m & =-\int_{\mathbb{R}^{2}} v_{1} f d v-E_{2} B .
\end{aligned}
$$

We then integrate in $x$ and use the boundary conditions (1.5)-(1.8)

$$
\begin{aligned}
& \frac{d}{d t}\left[\int_{0}^{1} \int_{\mathbb{R}^{2}} \sqrt{1+|v|^{2}} f(t, x, v) d x d v+\frac{1}{2} \int_{0}^{1}|\mathbf{E}(t, x)|^{2}+|B(t, x)|^{2} d x\right] \\
& -\int_{\left\{v_{1}<0\right\}} v_{x} f(t, 0, v) d v+\int_{\left\{v_{1}>0\right\}} v_{1} f(t, 1, v) d v \leq \int_{\left\{v_{1}>0\right\}} v_{1} g(t, v) .
\end{aligned}
$$

Finally, the density estimate allows us to reach our conclusion.

Lemma 2.2. Consider a smooth solution of the Vlasov equation (1.1) on the time interval $[0, T]$. Then, the electromagnetic fields are bounded in $L^{\infty}$ and

$$
\|\mathbf{E}\|_{\infty}+\|B\|_{\infty} \leq C_{1}
$$

where $C_{1}$ depends on the data $f_{0}, g, \mathbf{E}(0), B(0)$ and $T$. 
Proof. The key point of this proof is to get an $L^{\infty}$ bound on the electromagnetic fields that only depends on the natural estimates (2.2). We first find a modified Poisson equation to $E_{1}$ and next write $\left(E_{2}, B\right)$ as the solution of a one-dimensional wave equation.

From the Ampère equation (1.2) and the charge density conservation

$$
\frac{\partial \rho}{\partial t}+\frac{\partial j_{1}}{\partial x}=0
$$

we know that

$$
\frac{\partial E_{1}}{\partial x}(t, x)=E_{0}^{\prime}(x)-\rho(0, x)+\rho(t, x)
$$

which means that

$$
E_{1}(t, x)=-\int_{0}^{t} j_{1}(\tau, 0) d \tau+E_{1,0}(x)+\int_{0}^{x}(\rho(t, y)-\rho(0, y)) d y .
$$

But, from the energy estimate (2.1), the right-hand side is bounded. We then conclude that there exists a constant $C$ only depending on $T, f_{0}$ and $g$ such that

$$
\left\|E_{1}\right\|_{\infty} \leq C \text {. }
$$

Next we derive uniform bounds for the other field components $E_{2}$ and $B$. To this aim we apply the same argument as the one proposed by Glassey and Schaeffer in [8]. For this purpose we set

$$
k^{ \pm}(t, x)=E_{2}(t, x) \pm B(t, x),
$$

which satisfy the following transport equations

$$
\frac{\partial k^{ \pm}}{\partial t} \pm \frac{\partial k^{ \pm}}{\partial x}=-j_{2}(t, x)
$$

with initial condition

$$
k^{ \pm}(0, x)=k_{0}^{ \pm}(x)=E_{2,0}(x) \pm B_{0}(x) .
$$

Then the solution $k^{+}$can be written as

$$
k^{+}(t, x)= \begin{cases}k_{0}^{+}(x-t)-\int_{0}^{t} j_{2}(\tau, x-t+\tau) d \tau, & \text { if } x-t \geq 0, \\ k^{+}(t-x, 0)-\int_{t-x}^{t} j_{2}(\tau, x-t+\tau) d \tau, & \text { if } x-t \leq 0,\end{cases}
$$

and $k^{-}$is

$$
k^{-}(t, x)= \begin{cases}k_{0}^{-}(x+t)-\int_{0}^{t} j_{2}(\tau, x+t-\tau) d \tau, & \text { if } x+t \leq 1, \\ k^{-}(t+x, 1)-\int_{t+x-1}^{t} j_{2}(\tau, x+t-\tau) d \tau, & \text { if } x+t \geq 1 .\end{cases}
$$

Then, we have to estimate the following quantities:

$$
\int_{\alpha}^{t} j_{2}(\tau, x-t+\tau) d \tau, \quad \alpha \in\{0, t-x\}, \quad \int_{\beta}^{t} j_{2}(\tau, x+t-\tau) d \tau, \quad \beta \in\{0, t+x-1\}
$$

and

$$
k^{+}(s, 0), \quad k^{-}(s, 1), \quad s \in[0, T] .
$$

Let us start with (2.8). By the definitions of $e$ and $m$, we know that

$$
0 \leq e \pm m=\frac{1}{2}\left|E_{1}\right|^{2}+\frac{1}{2}\left(E_{2} \pm B\right)^{2}+\int_{\mathbb{R}^{2}}\left(\sqrt{1+|v|^{2}} \pm v_{1}\right) f d v .
$$


We integrate this equality over a backward characteristic triangle with vertex $(t, x)$ :

$$
y-x= \pm(\tau-t) .
$$

For $x-t \geq 0$, we deduce that

$$
\int_{0}^{t}(e+m)(\tau, x-t+\tau) d \tau=\int_{x-t}^{x} e(0, y) d y+\int_{0}^{t} m(\tau, x) d \tau,
$$

and when $x-t \leq 0$, we obtain

$$
\int_{t-x}^{t}(e+m)(\tau, x-t+\tau) d \tau=\int_{0}^{x} e(t-x, y) d y+\int_{t-x}^{t} m(\tau, x) d \tau .
$$

Next, when $x+t \leq 1$, we get

$$
\int_{0}^{t}(e-m)(\tau, x+t-\tau) d \tau=\int_{x}^{x+t} e(0, y) d y-\int_{0}^{t} m(\tau, x) d \tau
$$

and finally when $x+t \geq 1$,

$$
\int_{t+x-1}^{t}(e-m)(\tau, x+t-\tau) d \tau=\int_{x}^{1} e(t+x-1, y) d y-\int_{t+x-1}^{t} m(\tau, x) d \tau .
$$

To proceed, we split into four cases (see Fig. 1):

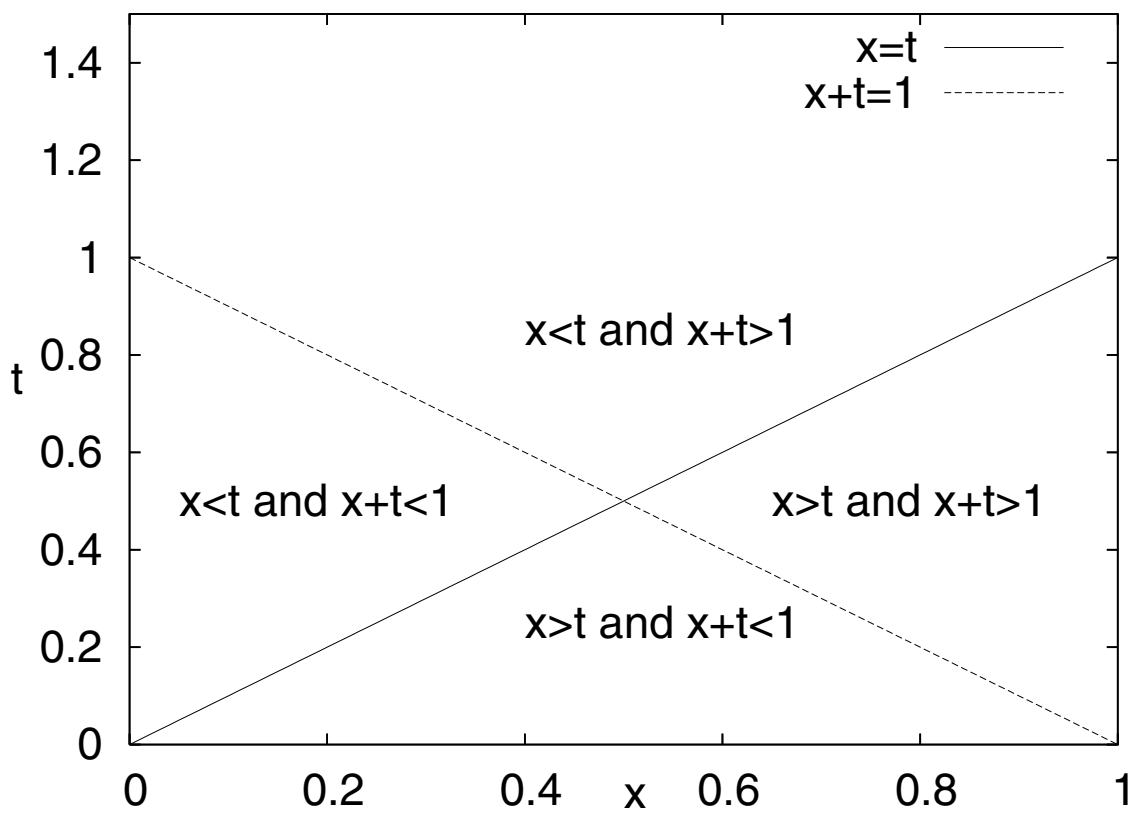

FIG. 1. Space-time picture of the different cases 
Case 1: $x-t \geq 0$ and $x+t \leq 1$. In this case, by adding the two equalities we get

$$
0 \leq \int_{0}^{t}(e+m)(\tau, x-t+\tau)+\int_{0}^{t}(e-m)(\tau, x+t-\tau) d \tau=\int_{x-t}^{x+t} e(0, y) d y
$$

and the right-hand side is uniformly bounded from the energy estimate (2.1).

Case 2: $x-t \leq 0$ and $x+t \leq 1$. Thus, we have

$$
\begin{array}{r}
0 \leq \int_{t-x}^{t}(e+m)(\tau, x-t+\tau)+\int_{0}^{t}(e-m)(\tau, x+t-\tau) d \tau \\
=\int_{0}^{x+t} e(0, y) d y-\int_{0}^{t-x} m(\tau, 0) d \tau .
\end{array}
$$

The first term on the right-hand side is of course bounded, whereas the second term satisfies (from the energy estimate (2.10)

$$
\begin{aligned}
-\int_{0}^{t-x} m(\tau, 0) d \tau & =\int_{0}^{t-x}\left[\int_{\mathbb{R}^{2}} v_{x} f(\tau, 0, v) d v+E_{y}(\tau, 0) B_{z}(\tau, 0)\right] d \tau \\
& \leq \int_{0}^{t-x} \int_{v_{x}>0} v_{x} f(\tau, 0, v) d v d \tau \leq C_{T},
\end{aligned}
$$

which gives the result.

Case 3: $x-t \geq 0$ and $x+t \geq 1$. In this case, we have

$$
\begin{array}{r}
0 \leq \int_{0}^{t}(e+m)(\tau, x-t+\tau) d \tau+\int_{t+x-1}^{t}(e-m)(\tau, x+t-\tau) d \tau \\
=\int_{x-t}^{1} e(0, y) d y+\int_{0}^{t+x-1} m(\tau, 1) d \tau
\end{array}
$$

and we proceed as in the previous case.

Case $4: x-t \leq 0$ and $x+t \geq 1$.

(a) If $t-x \leq t+x-1$, then we get

$$
\begin{array}{r}
0 \leq \int_{t-x}^{t}(e+m)(\tau, x-t+\tau) d \tau+\int_{t+x-1}^{t}(e-m)(\tau, x+t-\tau) d \tau \\
=\int_{0}^{1} e(t-x, y) d y+\int_{t-x}^{t+x-1} m(\tau, 1) d \tau .
\end{array}
$$

(b) If $t-x \geq t+x-1$, then we get

$$
\begin{array}{r}
0 \leq \int_{t-x}^{t}(e+m)(\tau, x-t+\tau) d \tau+\int_{t+x-1}^{t}(e-m)(\tau, x+t-\tau) d \tau \\
=\int_{0}^{1} e(t+x-1, y) d y-\int_{t+x-1}^{t-x} m(\tau, 0) d \tau .
\end{array}
$$

In both cases, we prove from the energy estimate that the right-hand side is bounded. Finally, using the crucial (the proof is elementary) inequality

$$
\frac{\left|v_{2}\right|}{\sqrt{1+|v|^{2}}} \leq \sqrt{1+|v|^{2}} \pm v_{1}
$$


we get the following result:

$$
\int_{t_{0}}^{t} \frac{\left|v_{2}\right|}{\sqrt{1+|v|^{2}}} f(\tau, x \pm t \mp \tau) d v d \tau \leq \int_{t_{0}}^{t}(e \pm m)(\tau, x \pm t \mp \tau) d \tau,
$$

and from (2.11)- 2.15), we conclude that

$$
\sup _{t, x} \int_{t_{0}}^{t} \frac{\left|v_{2}\right|}{\sqrt{1+|v|^{2}}} f(\tau, x \pm t \mp \tau) d v d \tau \leq C_{T}
$$

which gives a uniform bound for (2.8).

Now, let us deal with (2.9). We observe from the boundary conditions (1.8) that

$$
k^{+}(t, 0)=B(t, 0)=-k^{-}(t, 0)
$$

but for $t \leq 1$, we have

$$
k^{-}(t, 0)=k_{0}^{-}(t)-\int_{0}^{t} j_{2}(\tau, t-\tau) d \tau
$$

which can be uniformly estimated using (2.16). A similar argument holds true to estimate $k^{-}(t, 1)$.

Now, we proceed by induction and assume that $k^{ \pm}(t, 0)$ and $k^{ \pm}(t, 1)$ are uniformly bounded for $n-1 \leq t \leq n$. Thus, let us show that when $n \leq t \leq n+1, k^{ \pm}(t, 0)$ is bounded. We write $k^{ \pm}(t, 0)$ using the backward characteristic curves

$k^{+}(t, 0)=-k^{-}(t, 0)=-k^{-}(s, t-s)+\int_{s}^{t} j_{2}(\tau, t-\tau) d \tau, \quad n-1 \leq s \leq n, 0 \leq t-s \leq 1$.

The right-hand side is uniformly bounded since $k^{ \pm}(s, x)$ is bounded for $n-1 \leq s \leq n$ and $x \in[0,1]$.

Finally, the initial datum $k^{ \pm, 0}$ is bounded in $L^{\infty}$. Therefore, $k^{+}$and $k^{-}$are bounded in $L^{\infty}$, and we conclude that there exists a constant $C$ only depending on $T, f_{0}$ and $g$ such that

$$
\|\mathbf{E}\|_{\infty}+\|B\|_{\infty} \leq C
$$

\section{2. $C^{0,1}$ estimate.}

LEMma 2.3. Consider a smooth solution of the Vlasov equation (1.1), which is compactly supported on the time interval $[0, T]$. Then, the electromagnetic fields are bounded in $C^{0,1}$ and

$$
\|\mathbf{E}\|_{C^{0,1}}+\|B\|_{C^{0,1}} \leq C_{2}
$$

where $C_{2}$ depends on the support of $f$ in $v$.

Proof. Now, we need to estimate the derivatives of the fields. We start with $E_{1}$, which satisfies

$$
\frac{\partial E_{1}}{\partial x}=E_{1,0}^{\prime}(x)-\rho(0, x)+\rho(t, x), \quad \frac{\partial E_{1}}{\partial t}=-j_{1}(t, 0) .
$$

Assuming that $\rho$ is bounded in $L^{\infty}$, we get the result

$$
\left\|E_{1}\right\|_{C^{0,1}} \leq C R^{2} T
$$


where $R$ is an upper bound of the support of $f$ in $v$.

On the other hand, $\partial k^{+} / \partial t$ satisfies

$$
\frac{\partial k^{+}}{\partial x}(t, x)= \begin{cases}k_{0}^{+\prime}(x-t)-\int_{0}^{t} \frac{\partial j_{2}}{\partial x}(\tau, x-t+\tau) d \tau, & \text { if } x-t \geq 0, \\ -\frac{\partial k^{+}}{\partial t}(t-x, 0)-j_{2}(t-x, 0)-\int_{t-x}^{t} \frac{\partial j_{2}}{\partial x}(\tau, x-t+\tau) d \tau, & \text { if } x-t \leq 0 .\end{cases}
$$

To find an upper bound of the derivative, we apply the proof of [8] when the origin of the backward characteristic is inside the domain, whereas we proceed by induction using the backward characteristics when $\partial k^{+} / \partial x$ is given by the boundary value.

From [8, we introduce the differential operators

$$
T_{+}=\partial_{t}+\partial_{x}, \quad S=\partial_{t}+\hat{v}_{1}(v) \partial_{x}
$$

Then,

$$
\partial_{t}=\frac{S-\hat{v}_{1}(v) T_{+}}{1-\hat{v}_{1}(v)}, \quad \partial_{x}=\frac{T_{+}-S}{1-\hat{v}_{1}(v)} .
$$

We obtain for $\alpha=0$ or $t-x$,

$$
\begin{aligned}
\int_{\alpha}^{t} \frac{\partial j_{2}}{\partial x}(\tau, x-t+\tau) d \tau & =\int_{\alpha}^{t} \int_{\mathbb{R}^{2}} \frac{\hat{v}_{2}(v)}{1-\hat{v}_{1}(v)}\left(T_{+} f-S f\right)(\tau, x-t+\tau, v) d v d \tau \\
& =\int_{\alpha}^{t} \frac{d}{d \tau} \int_{\mathbb{R}^{2}} \frac{\hat{v}_{2}(v)}{1-\hat{v}_{1}(v)} f(\tau, x-t+\tau, v) d v d \tau \\
& +\int_{\alpha}^{t} \int_{\mathbb{R}^{2}} \frac{\hat{v}_{2}(v)}{1-\hat{v}_{1}(v)} \nabla_{v} \cdot(\mathbf{E}+\hat{v} \times \mathbf{B}) f(\tau, x-t+\tau, v) d v d \tau
\end{aligned}
$$

where we have used the Vlasov equation in the last term. Now since $f$ is compactly supported we get

$$
\begin{aligned}
\int_{\alpha}^{t} \frac{\partial j_{2}}{\partial x}(\tau, x-t+\tau) d \tau & =\int_{\mathbb{R}^{2}} \frac{\hat{v}_{2}(v)}{1-\hat{v}_{1}(v)} f(t, x, v) d v-\int_{\mathbb{R}^{2}} \frac{\hat{v}_{2}(v)}{1-\hat{v}_{1}(v)} f(\alpha, x-t+\alpha, v) d v \\
& -\int_{\alpha}^{t} \int_{\mathbb{R}^{2}} \nabla_{v}\left(\frac{\hat{v}_{2}(v)}{1-\hat{v}_{1}(v)}\right) \cdot(\mathbf{E}+\hat{v} \times \mathbf{B}) f(\tau, x-t+\tau, v) d v d \tau .
\end{aligned}
$$

Each integral is taken on a bounded domain $B(0, R)$, and hence there exists $C_{T}$ such that

$$
\frac{1}{1-\hat{v}_{1}(v)} \leq C_{T}, \quad 0 \leq t \leq T .
$$

A similar bound holds for the gradient of $\hat{v}_{2}(v) /\left(1-\hat{v}_{1}(v)\right)$. Since both $f$ and $(\mathbf{E}, B)$ are bounded in $L^{\infty}$, this leads to

$$
\left\|\int_{\alpha}^{t} \frac{\partial j_{2}}{\partial x}(\tau, x-t+\tau) d \tau\right\|_{\infty} \leq C_{T} .
$$

Finally, we treat similarly

$$
\int_{\alpha}^{t} \frac{\partial j_{2}}{\partial x}(\tau, x+t-\tau) d \tau
$$


and use the characteristics as previously to estimate the boundary value for $x=0$ and $x=1$ leading to the result

$$
\left\|\frac{\partial k^{+}}{\partial x}\right\|_{\infty}+\left\|\frac{\partial k^{-}}{\partial x}\right\|_{\infty} \leq C_{T}
$$

3. Distribution function estimates. In this section, we consider the linear VlasovMaxwell equation with a smooth $C^{0,1}$ external electromagnetic field. We first prove that the characteristic curves corresponding to the particle trajectories can be correctly defined. Then, we approximate the Vlasov equation by a transport equation with source term giving a unique $C^{1,0}$ smooth solution. For this latter problem we finally get a uniform $B V$ estimate.

3.1. Particle trajectories. We consider the following linear problem with a given, external electromagnetic field $\mathbf{E}=\left(E_{1}, E_{2}, 0\right), \mathbf{B}=(0,0, B)$ :

$$
\frac{\partial f}{\partial t}+\hat{v}_{1}(v) \frac{\partial f}{\partial x}+(\mathbf{E}+\hat{v}(v) \times \mathbf{B}) \cdot \nabla_{v} f=0, \quad t \in[0, T], x \in(0,1), v \in \mathbb{R}^{2},
$$

with the initial datum

$$
f(0, x, v)=f_{0}(x, v), \quad x \in(0,1), v \in \mathbb{R}^{2}
$$

and boundary conditions

$$
\left.f\right|_{\gamma_{0}^{+}}=g,\left.\quad f\right|_{\gamma_{1}^{-}}=0
$$

For any point $(t, x, v) \in \Pi$, we define

$$
\Gamma(\tau, t, x, v)=(\tau, X(\tau, t, x, v), V(\tau, t, x, v))
$$

to be the unique trajectory of

$$
\left\{\begin{array}{l}
\frac{d X}{d \tau}(\tau)=\hat{v}_{1}(V(\tau)) \\
\frac{d V_{x}}{d \tau}(\tau)=E_{1}(\tau, X(\tau))+\hat{v}_{2}(V(\tau)) B(\tau, X(\tau)) \\
\frac{d V_{y}}{d \tau}(\tau)=E_{2}(\tau, X(\tau))-\hat{v}_{1}(V(\tau)) B(\tau, X(\tau)),
\end{array}\right.
$$

such that $X(t, t, x, v)=x, V(t, t, x, v)=v$. Then, equivalently, the solution can be written as

$$
\begin{aligned}
X(s) & =x+\int_{t}^{s} \hat{v}_{1}(V(\tau)) d \tau, \\
V(s) & =v+\int_{t}^{s} \mathbf{E}(\tau, X(\tau))+\hat{v}(V(\tau)) \times \mathbf{B}(\tau, X(\tau)) d \tau .
\end{aligned}
$$

We define the origin of $\left(t, x, v_{1}, v_{2}\right)$ by $\left(t_{0}, x_{0}, v_{0,1}, v_{0,2}\right)$, which is the (unique) first point at $\partial \Pi$ on the backward-in-time trajectory (3.2). We finally define the differential operator 
$D$ by

$$
D F\left(x, v_{x}, v_{y}\right)=\left(\begin{array}{c}
\partial_{x} F \\
\partial_{v_{x}} F \\
\partial_{v_{y}} F
\end{array}\right) .
$$

We begin with two basic facts for such a starting point $\left(t_{0}, x_{0}, v_{0,1}, v_{0,2}\right)$.

Lemma 3.1. Let $\mathbf{E}, B \in C^{1}([0, T] \times[0,1])$ and $\left(t, x, v_{1}, v_{2}\right) \in \Pi$ and assume that $t_{0}=0$. Then its origin points $\left(x_{0}^{(1)}, v_{0,1}^{(1)}, v_{0,2}^{(1)}\right)$ are $C^{1}$ functions near $\left(t, x, v_{1}, v_{2}\right)$ and

$$
\begin{aligned}
x_{0}^{(1)} & =x-\int_{0}^{t} \hat{v}_{1}(V(\tau)) d \tau, \\
v_{0}^{(1)} & =v-\int_{0}^{t}\{\mathbf{E}(\tau, X(\tau))+\hat{v}(V(\tau)) \times \mathbf{B}(\tau, X(\tau))\} d \tau .
\end{aligned}
$$

Moreover,

$$
\left\{\begin{array}{l}
\frac{\partial x_{0}^{(1)}}{\partial t}=-\hat{v}_{1}(v)-\int_{0}^{t} D \hat{v}_{1}(V(\tau)) \cdot \Gamma_{t}(\tau, t, x, v) d \tau \\
\frac{\partial x_{0}^{(1)}}{\partial x}=1-\int_{0}^{t} D \hat{v}_{1}(V(\tau)) \cdot \Gamma_{x}(\tau, t, x, v) d \tau \\
\nabla_{v} x_{0}^{(1)}=-\int_{0}^{t} D \hat{v}_{1}(V(\tau)) \cdot \nabla_{v} \Gamma(\tau, t, x, v) d \tau
\end{array}\right.
$$

and

$$
\left\{\begin{aligned}
\frac{\partial v_{0}^{(1)}}{\partial t}= & -\mathbf{E}(t, x)-\hat{v}(v) \times \mathbf{B}(t, x) \\
& -\int_{0}^{t} D(\mathbf{E}(\tau)-\hat{v} \times \mathbf{B}(\tau)) \cdot \Gamma_{t}(\tau, t, x, v) d \tau, \\
\frac{\partial v_{0}^{(1)}}{\partial x}= & -\int_{0}^{t} D(\mathbf{E}(\tau)-\hat{v} \times \mathbf{B}(\tau)) \cdot \Gamma_{x}(\tau, t, x, v) d \tau, \\
\nabla_{v} v_{0}^{(1)}= & I d_{2}-\int_{0}^{t} D(\mathbf{E}(\tau)-\hat{v} \times \mathbf{B}(\tau)) \cdot \nabla_{v} \Gamma(\tau, t, x, v) d \tau .
\end{aligned}\right.
$$

Now we consider the situation where the origin of the characteristic is at $x_{0}=0$.

Lemma 3.2. Let $\mathbf{E}, B \in C^{1}([0, T] \times[0,1])$ and fix $\left(t, x, v_{1}, v_{2}\right) \in \Pi$ such that $x_{0}=0$ and $\hat{v}_{1}\left(v_{0}^{(2)}\right)>0$. Then $\left(t_{0}^{(2)}, v_{0,1}^{(2)}, v_{0,2}^{(2)}\right)$ are $C^{1}$ functions near $\left(t, x, v_{1}, v_{2}\right)$ and

$$
\begin{aligned}
x & =\int_{t_{0}^{(2)}}^{t} \hat{v}_{1}(V(\tau)) d \tau, \\
v_{0}^{(2)} & =v-\int_{t_{0}^{(2)}}^{t} \mathbf{E}(\tau, X(\tau))-\hat{v}(V(\tau)) \times \mathbf{B}(\tau, X(\tau)) d \tau .
\end{aligned}
$$


Moreover,

$$
\left\{\begin{array}{l}
\frac{\partial t_{0}^{(2)}}{\partial t}=\frac{1}{\hat{v}_{1}\left(v_{0}^{(2)}\right)}\left(\hat{v}_{1}(v)+\int_{t_{0}^{(2)}}^{t} D \hat{v}_{1}(V(\tau)) \cdot \Gamma_{t}(\tau, t, x, v) d \tau\right) \\
\frac{\partial t_{0}^{(2)}}{\partial x}=\frac{1}{\hat{v}_{1}\left(v_{0}^{(2)}\right)}\left(-1+\int_{t_{0}^{(2)}}^{t} D \hat{v}_{1}(V(\tau)) \cdot \Gamma_{x}(\tau, t, x, v) d \tau\right) \\
\nabla_{v} t_{0}^{(2)}=\frac{1}{\hat{v}_{1}\left(v_{0}^{(2)}\right)}\left(\int_{t_{0}^{(2)}}^{t} D \hat{v}_{1}(V(\tau)) \cdot \nabla_{v} \Gamma(\tau, t, x, v) d \tau\right)
\end{array}\right.
$$

and

$$
\left\{\begin{aligned}
\frac{\partial v_{0}^{(2)}}{\partial t}= & \left(\mathbf{E}\left(t_{0}^{(2)}, 0\right)+\hat{v}\left(v_{0}^{(2)}\right) \times \mathbf{B}\left(t_{0}^{(2)}, 0\right)\right) \frac{\partial t_{0}^{(2)}}{\partial t}-\mathbf{E}(t, x)-\hat{v}(v) \times \mathbf{B}(t, x) \\
& -\int_{t_{0}^{(2)}}^{t} D(\mathbf{E}(\tau)-\hat{v} \times \mathbf{B}(\tau)) \cdot \Gamma_{t}(\tau, t, x, v) d \tau \\
\frac{\partial v_{0}^{(2)}}{\partial x}= & \left(\mathbf{E}\left(t_{0}^{(2)}, 0\right)+\hat{v}\left(v_{0}^{(2)}\right) \times \mathbf{B}\left(t_{0}^{(2)}, 0\right)\right) \frac{\partial t_{0}^{(2)}}{\partial x} \\
& -\int_{t_{0}^{(2)}}^{t} D(\mathbf{E}(\tau)-\hat{v} \times \mathbf{B}(\tau)) \cdot \Gamma_{x}(\tau, t, x, v) d \tau \\
\nabla_{v} v_{0}^{(2)}= & I d_{2}+\left(\mathbf{E}\left(t_{0}^{(2)}, 0\right)+\hat{v}\left(v_{0}^{(2)}\right) \times \mathbf{B}\left(t_{0}^{(2)}, 0\right)\right) \nabla_{v} t_{0}^{(2)} \\
& -\int_{t_{0}^{(2)}}^{t} D(\mathbf{E}(\tau)+\hat{v} \times \mathbf{B}(\tau)) \cdot \nabla_{v} \Gamma(\tau, t, x, v) d \tau
\end{aligned}\right.
$$

Proof. We consider a $C^{1}$ extension $\overline{\mathbf{E}}$ and $\bar{B}$ of $\mathbf{E}$ and $B$ on the whole line $-\infty<x<\infty$ so that

$$
\|\bar{B}\|_{C^{1}} \leq C\|B\|_{C^{1}}, \quad\left\|\bar{E}_{\alpha}\right\|_{C^{1}} \leq C\left\|E_{\alpha}\right\|_{C^{1}}, \quad \alpha \in\{1,2\} .
$$

We consider (3.3)-(3.4) with such new $\overline{\mathbf{E}}$ and $\bar{B}$. We fix $\left(t, x, v_{1}, v_{2}\right) \in \Pi$ and from our assumption, we have

$$
\frac{\partial X}{\partial s}\left(s=t_{0}^{(2)}, t, x, v_{1}, v_{2}\right)=\hat{v}_{1}\left(v_{0}^{(2)}\right)>0 .
$$

Moreover, $\overline{\mathbf{E}}$ and $\bar{B}$ are smooth functions of $\left(t, x, v_{1}, v_{2}\right)$, and then the solution to (3.3)(3.4) is $C^{1}$. From these statements, we can apply the Implicit Function Theorem to find a unique $C^{1}$ function $\theta\left(t, x, v_{1}, v_{2}\right)$ locally, which satisfies

$$
X\left(\theta\left(t, x, v_{1}, v_{2}\right), t, x, v_{1}, v_{2}\right)=0, \quad t_{0}=\theta\left(t, x, v_{1}, v_{2}\right)
$$

and for $\left(t^{\prime}, x^{\prime}, v_{1}^{\prime}, v_{2}^{\prime}\right)$ close to $\left(t, x, v_{1}, v_{2}\right)$,

$$
X\left(\theta\left(t^{\prime}, x^{\prime}, v_{1}^{\prime}, v_{2}^{\prime}\right), t^{\prime}, x^{\prime}, v_{1}^{\prime}, v_{2}^{\prime}\right)=0 .
$$

Now we want to show that for any $\left(t^{\prime}, x^{\prime}, v_{1}^{\prime}, v_{2}^{\prime}\right)$ near $\left(t, x, v_{1}, v_{2}\right)$,

$$
X\left(s, t^{\prime}, x^{\prime}, v^{\prime}\right)>0, \quad \theta\left(t^{\prime}, x^{\prime}, v^{\prime}\right)<s \leq t^{\prime},
$$

which will imply that $\bar{E}=E$ and $\bar{B}=B$. 
On the one hand, since for $\left(t^{\prime}, x^{\prime}, v_{1}^{\prime}, v_{2}^{\prime}\right)$ close to $\left(t, x, v_{1}, v_{2}\right)$, the function $t_{0}^{(2)}$ belongs to $C^{1}$ and

$$
\frac{\partial X}{\partial s}\left(s=\theta\left(t, x, v_{1}, v_{2}\right), t, x, v_{1}, v_{2}\right)=v_{0}^{(2)}>0,
$$

it follows that there is a $\delta>0$ such that

$$
X\left(s, t^{\prime}, x^{\prime}, v^{\prime}\right)>0, \quad \theta\left(t^{\prime}, x^{\prime}, v_{1}^{\prime}, v_{2}^{\prime}\right)<s<\theta\left(t^{\prime}, x^{\prime}, v_{1}^{\prime}, v_{2}^{\prime}\right)+\delta .
$$

On the other hand, by the definition of $t_{0}^{(2)}$, which is the smallest time such that

$$
X\left(t_{0}^{(2)}, t, x, v_{1}, v_{2}\right)=0 \text { and } X\left(t, t, x, v_{1}, v_{2}\right)=x>0,
$$

there exists $\epsilon>0$ such that

$$
X\left(s, t, x, v_{1}, v_{2}\right) \geq \epsilon>0, \quad t_{0}^{(2)}+\delta / 2 \leq s \leq t .
$$

It follows that by the continuity of $X$ with respect to $\left(t, x, v_{1}, v_{2}\right)$ and by further choosing $\left(t^{\prime}, x^{\prime}, v_{1}^{\prime}, v_{2}^{\prime}\right)$ closer to $\left(t, x, v_{1}, v_{2}\right)$,

$$
X\left(s, t^{\prime}, x^{\prime}, v_{1}^{\prime}, v_{2}^{\prime}\right) \geq \epsilon_{0} / 2>0, \quad t_{0}+\delta \leq s \leq t^{\prime} .
$$

Therefore, since $\theta\left(t, x, v_{1}, v_{2}\right)=t_{0}$, gathering (3.11) and (3.12) we get

$$
X\left(s, t^{\prime}, x^{\prime}, v_{1}^{\prime}, v_{2}^{\prime}\right)>0, \quad \theta\left(t^{\prime}, x^{\prime}, v^{\prime}\right)<s \leq t^{\prime}
$$

and $\theta\left(t^{\prime}, x^{\prime}, v^{\prime}\right)=t_{0}^{(2)}\left(t^{\prime}, x^{\prime}, v^{\prime}\right)$. Finally, (3.8) and (3.9) follow by differentiating (3.3) and (3.4).

3.2. Approximated problem. We first define a regularized approximation to (1.1) with initial and boundary conditions (1.5)-(1.6) with a damping term. Let $r$ be such that

$$
r=\left(x^{2}(1-x)^{2}+v_{1}^{2}\right)^{1 / 2}
$$

and consider the problem

$$
\frac{\partial f}{\partial t}+\hat{v}_{1}(v) \frac{\partial f}{\partial x}+(\mathbf{E}+\hat{v}(v) \times \mathbf{B}) \cdot \nabla_{v} f=-\varepsilon r^{-1-\alpha} f, \quad x \in(0,1), v \in \mathbb{R}^{2},
$$

with $\varepsilon>0,1>\alpha>0$ and smooth electromagnetic $\mathbf{E}$ and $\mathbf{B}$ given by (1.2)-(1.4) with a regularized initial datum

$$
f_{0}^{\varepsilon}(x, v)=f_{0} \star \theta_{\varepsilon}(x, v)
$$

with suitable mollifier $\theta_{\varepsilon}$ such that $f_{0}^{\varepsilon}(x, v) \equiv 0$ for $x$ near both $x=0$ and $x=1$. Using the same technique we also construct $g^{\varepsilon}$ such that

$$
g^{\varepsilon}(t, v) \equiv 0
$$

for $(t, x)$ near either $t=0$ or $v=0$. It is important to note that both $f_{0}^{\varepsilon}$ and $g^{\varepsilon}$ can be chosen to have uniform $B V$ bounds with respect to $\varepsilon$.

In this section we always assume that the electromagnetic fields are given. Thus, we first establish the $C^{1}$ estimate for the approximate linear problem. Finally, we establish a $B V$ estimate to (3.13) uniformly with respect to $\varepsilon$ and prove the convergence to the solution to (1.1) when $\varepsilon$ goes to zero.

We are now ready to establish the $C^{1}$ estimate for the approximate linear problem. 
Lemma 3.3. Let $E, B$ be $C^{1}([0, T] \times[0,1])$. Let $f_{0}^{\varepsilon}$ and $g^{\varepsilon}$ be $C^{1}$. Then the solution $f^{\varepsilon}\left(t, x, v_{1}, v_{2}\right)$ to the linear approximate Vlasov equation (3.13) with boundary conditions (1.5) and (1.6) belongs to $C^{1}(\Pi)$.

Proof. For any $\left(t, x, v_{x}, v_{y}\right) \in \Pi \backslash \gamma_{S}$ we consider its backward trajectory $\Gamma\left(\tau, t, x, v_{1}, v_{2}\right)$ to (3.2) with respect to the external fields $\mathbf{E}(t, x), B(t, x)$ for $0 \leq \tau \leq t$. $\Gamma$ can emanate from different parts of the boundary of $\Pi$.

(a) If $\Gamma$ emanates from $\Pi_{0}$, then we define

$$
f^{\varepsilon}\left(t, x, v_{1}, v_{2}\right)=f_{0}\left(x_{0}^{(1)}, v_{0,1}^{(1)}, v_{0,2}^{(1)}\right) \exp \left(-\varepsilon \int_{0}^{t} r^{-1-\alpha}(\tau) d \tau\right),
$$

where $r(\tau)=r\left(\Gamma\left(\tau, t, x, v_{x}, v_{y}\right)\right)$.

(b) If $\Gamma$ emanates from $\gamma_{0}^{+}$, then we define

$$
f^{\varepsilon}\left(t, x, v_{x}, v_{y}\right)=g\left(t_{0}^{(2)}, v_{0,1}^{(2)}, v_{0,2}^{(2)}\right) \exp \left(-\varepsilon \int_{t_{0}^{(2)}}^{t} r^{-1-\alpha}(\tau) d \tau\right) .
$$

(c) If $\Gamma$ emanates from $\gamma_{1}^{-}$or $\gamma_{1}^{0}$ or $\gamma_{0}^{0}$, then we define

$$
f^{\varepsilon}\left(t, x, v_{1}, v_{2}\right)=0 \text {. }
$$

Thanks to our construction of $f_{0}^{\varepsilon}$ and $g^{\varepsilon}$, the three expressions for $f$ are zero at $\Pi_{0} \cap \gamma_{0}^{+}$ and $\Pi \cap \gamma_{1}^{-}$. Moreover since $f_{0}^{\varepsilon}$ vanishes near $\gamma_{0}^{0}$ and $\gamma_{1}^{0}$, the first expression is compatible with the last one when $\left(t_{0}, x_{0}, v_{0,1}\right)=(0,0,0)$ and $\left(t_{0}, x_{0}, v_{1,0}\right)=(0,1,0)$.

Now, we prove that $f$ is $C^{1}$. Since the domains of definition of $f$ have nonempty intersections, different cases can occur.

Case 1. $\Gamma$ emanates from $\Pi_{0}$ with $0<x_{0}<1$ or from $\gamma_{0}^{+}$with $t_{0}>0$, or from $\gamma_{1}^{-}$with $t_{0}>0$. In these cases we have only one expression for $f$. Then by standard ODE theory, we easily deduce that $f \in C^{1}$ near such a point $\left(t, x, v_{1}, v_{2}\right)$.

Case 2. $\Gamma$ emanates from $\Pi_{0} \cap \gamma_{0}^{+}=\left\{t=0, x=0, v_{1}>0\right\}$ or $\Pi_{0} \cap \gamma_{1}^{-}=\{t=0, x=$ $\left.1, v_{1}<0\right\}$. From our construction of $f_{0}^{\varepsilon}$ and $g^{\varepsilon}$, we deduce that $f^{\varepsilon}$ vanishes identically near $\Gamma$. Hence $f^{\varepsilon}$ is $C^{\infty}$ in this case.

Case 3. $\Gamma$ emanates from singular sets $\gamma_{0}^{0}$ or $\gamma_{1}^{0}$. We have

$$
f^{\varepsilon}\left(t, x, v_{1}, v_{2}\right)=0 \text {. }
$$

To prove that $f^{\varepsilon}$ is $C^{1}$ at this point it suffices to show that $f^{\varepsilon}$ vanishes exponentially when $\left(t^{\prime}, x^{\prime}, v_{1}^{\prime}, v_{2}^{\prime}\right)$ goes to $\left(t, x, v_{1}, v_{2}\right)$. Since the flow is $C^{1}$, it is enough to prove that $f$ vanishes exponentially fast only when $\left(t, x, v_{1}, v_{2}\right) \in \gamma_{0}^{0}$. Moreover, by definition of $r$,

$$
r\left(t, x, v_{1}\right)=\left(x^{2}(1-x)^{2}+v_{1}^{2}\right)^{1 / 2},
$$

we just have to prove that $f^{\varepsilon}\left(t, x, v_{1}, v_{2}\right)$ tends exponentially fast to zero when $r$ goes to zero.

Recall that the trajectories are bounded. Hence for a given $T>0$ and $t<T$, there exists a uniform bound $C_{T}$ for $\hat{v}_{x}(v)$ and $\mathbf{E}(t, x)+\hat{v}(v) \times \mathbf{B}(t, x)$ when $\left(t, x, v_{1}, v_{2}\right)$ is in the support of $f^{\varepsilon}$ and we can write

$$
f^{\varepsilon}\left(t, x, v_{1}, v_{2}\right)=f^{\varepsilon}\left(t_{0}, x_{0}, v_{1,0}, v_{2,0}\right) \exp \left(-\varepsilon \int_{t_{0}}^{t} r^{-1-\alpha}(\tau) d \tau\right)
$$


where

$$
f^{\varepsilon}\left(t_{0}, x_{0}, v_{x, 0}, v_{y, 0}\right)= \begin{cases}f_{0}^{\varepsilon}\left(x_{0}, v_{1,0}, v_{2,0}\right), & \text { if } t_{0}=0, \\ g^{\varepsilon}\left(t_{0}, v_{1,0}, v_{2,0}\right), & \text { if } x_{0}=0, \\ 0, & \text { if } x_{0}=1 \text { or }\left(t_{0}, x_{0}, v_{0,1}\right)=(0,0,0) .\end{cases}
$$

If $\left(t, x, v_{1}, v_{2}\right)$ is in the support of $f$, then $\Gamma(\tau)$ is of course also in the support of $f$ and we can write

$$
\left|\frac{d r}{d \tau}(t)\right| \leq C
$$

so that

$$
\left|r(t)-r\left(t_{0}\right)\right| \leq C\left|t-t_{0}\right|
$$

Then two cases occur:

(i) $\left|t_{0}-t\right| \leq r(t) / C$. Then

$$
\begin{aligned}
r\left(t_{0}\right) & \leq r(t)+C\left|t-t_{0}\right| \\
& \leq 2 r(t) .
\end{aligned}
$$

Thus, if further

$$
r(t) \leq \varepsilon / 2,
$$

then $f^{\varepsilon}\left(t_{0}, x_{0}, v_{1,0}, v_{2,0}\right)=0$ by the property of $f_{0}^{\varepsilon}$ and $g^{\varepsilon}$. Hence $f^{\varepsilon}\left(t, x, v_{1}, v_{2}\right)=$ 0 .

(ii) $\left|t_{0}-t\right|>r(t) / C$. Then

$$
-\varepsilon \int_{t_{0}}^{t} r^{-1-\alpha}(\tau) d \tau \leq-\varepsilon \int_{t-r(t) / C}^{t} r^{-1-\alpha}(\tau) d \tau
$$

hence

$-\varepsilon \int_{t_{0}}^{t} r^{-1-\alpha}(\tau) d \tau \leq-\varepsilon \int_{t-r(t) / C}^{t} 2^{-1-\alpha} r^{-1-\alpha}(t) d \tau \leq-\varepsilon 2^{-1-\alpha} r^{-\alpha}(t) / C$

and

$$
f^{\varepsilon}\left(t, x, v_{1}, v_{2}\right) \leq f^{\varepsilon}\left(t_{0}, x_{0}, v_{0,1}, v_{0,2}\right) \leq \exp \left(-\varepsilon 2^{-1-\alpha} r^{-\alpha}(t) / C\right),
$$

which proves that $f^{\varepsilon}$ goes exponentially to zero when $r$ goes to zero.

3.3. BV estimate. Even for the simple Vlasov-Poisson problem the solution $f$ to (1.1) coupled with (1.2) and $B=0$ is not continuous in general; see [11. To characterize such a singularity, we now turn to a $B V$ estimate of discontinuous solutions to (1.1).

Proposition 3.4. Let $E, B$ be $C^{1}([0, T] \times[0,1])$. Assume $0 \leq g(t, v) \in B V \cap L^{\infty}\left(\gamma_{0}^{+}\right)$, $v \nabla_{v} g \in L^{1}\left(\gamma_{0}^{+}\right), 0 \leq f_{0}(x, v) \in B V \cap L^{\infty}\left(\Pi_{0}\right)$. Then there exists a unique solution to the linear Vlasov equation (1.1) that satisfies

$$
T V[f(t)] \leq C\left(\|\mathbf{E}\|_{C^{0,1}}+\|B\|_{C^{0,1}}, T\right)\left\{T V\left[f_{0}\right]+\left\|f_{0}\right\|_{\infty}+\int_{\gamma_{0}^{+}}[1+v]\left|\nabla_{v} g\right| d v d t+\|g\|_{\infty}\right\} .
$$


Proof. Let us introduce $\gamma_{0, s}^{+}$:

$$
\gamma_{0, s}^{+}=\left\{\left(t, 0, v_{1}, v_{2}\right), \quad v_{x} \geq 0,0 \leq t \leq s\right\} .
$$

Let $\varepsilon>0$ and $1>\alpha>0$. We construct regularized approximations such that $f_{0}^{\varepsilon}, g^{\varepsilon}$ are $C^{1}$ functions being compactly supported in $\left(v_{1}, v_{2}\right)$, as in (3.14). From these data, we construct $C^{1}$ solutions to (3.13). Consider $f_{\alpha}^{\varepsilon}$, the derivative of $f^{\varepsilon}$ with respect to $\alpha \in\left\{x, v_{1}, v_{2}\right\}$. We first differentiate the equation (3.13) with respect to $x$. Then

$$
\begin{aligned}
\frac{\partial f_{x}^{\varepsilon}}{\partial t} & +\hat{v}_{1}(v) \frac{\partial f_{x}^{\varepsilon}}{\partial x}+(\mathbf{E}+\hat{v}(v) \times \mathbf{B}) \cdot \nabla_{v} f_{x}^{\varepsilon} \\
& =-\varepsilon r^{-1-\alpha} f_{x}^{\varepsilon}-\left(\frac{\partial \mathbf{E}}{\partial x}+\hat{v}(v) \times \frac{\partial \mathbf{B}}{\partial x}\right) \cdot \nabla_{v} f^{\varepsilon}-\varepsilon \frac{\partial r^{-1-\alpha}}{\partial x} f^{\varepsilon}
\end{aligned}
$$

and

$$
\begin{aligned}
f_{x}^{\varepsilon}\left(0, x, v_{1}, v_{2}\right) & =\frac{\partial f_{0}^{\varepsilon}}{\partial x}\left(x, v_{1}, v_{2}\right), \\
f_{x}^{\varepsilon}\left(t, 1, v_{1}, v_{2}\right) & =0
\end{aligned}
$$

and for $v_{1}>0$,

$$
f_{x}^{\varepsilon}\left(t, 0, v_{1}, v_{2}\right)=-\frac{1}{\hat{v}_{1}(v)}\left(\frac{\partial g_{0}^{\varepsilon}}{\partial t}+(\mathbf{E}(t, 0)+\hat{v}(v) \times \mathbf{B}(t, 0)) \cdot \nabla_{v} g^{\varepsilon}-\varepsilon v_{x}^{-1-\alpha} g^{\varepsilon}\right) .
$$

We deduce that

$$
\begin{aligned}
& \int_{\Pi_{s}}\left|f_{x}^{\varepsilon}\right| d x d v-\int_{\gamma_{0, s}^{+}} \hat{v}_{1}(v)\left|f_{x}^{\varepsilon}\right| d t d v \\
& \quad=\int_{\Pi_{0}}\left|f_{x}^{\varepsilon}\right| d x d v \\
& \quad+\int_{\gamma_{0, s}^{+}} \frac{\hat{v}_{1}(v)}{\hat{v}_{1}(v) \mid}\left|\frac{\partial g^{\varepsilon}}{\partial t}+(\mathbf{E}(t, 0)+\hat{v}(v) \times \mathbf{B}(t, 0)) \cdot \nabla_{v} g^{\varepsilon}+\varepsilon v_{1}^{-1-\alpha} g^{\varepsilon}\right| d t d v \\
& \quad-\int_{0}^{s} \int_{\Pi_{\tau}} \operatorname{sign}\left(f_{x}^{\varepsilon}\right)\left[\varepsilon r^{-1-\alpha} f_{x}^{\varepsilon}+\left(\frac{\partial \mathbf{E}}{\partial x}+\hat{v}(v) \times \frac{\partial \mathbf{B}}{\partial x}\right) \cdot \nabla_{v} f^{\varepsilon}\right] d x d v d t \\
& \quad-\int_{0}^{s} \int_{\Pi_{\tau}} \operatorname{sign}\left(f_{x}^{\varepsilon}\right)\left[\varepsilon \frac{\partial r^{-1-\alpha}}{\partial x} f^{\varepsilon}\right] d x d v d t .
\end{aligned}
$$

We recall that $f_{0}^{\varepsilon}, g^{\varepsilon}$ are bounded in $W^{1,1}$, in the electromagnetic fields and $\hat{v}$ in $W^{1, \infty}$. Thus different terms can be estimated in the above equality and we get

$$
\begin{aligned}
& \int_{\Pi_{s}}\left|f_{x}^{\varepsilon}\right| d x d v-\int_{0}^{s} \int_{\gamma_{0, s}^{+}} \hat{v}_{x}(v)\left|f_{x}^{\varepsilon}\right| d t d v \\
& \quad \leq \quad C+C \int_{0}^{s} \int_{\Pi_{\tau}}\left(\left|f_{x}^{\varepsilon}\right|+\left|\nabla_{v} f^{\varepsilon}\right|\right) d x d v d t \\
& \quad+\varepsilon \int_{\gamma_{0, s}^{+}}\left|v_{1}\right|^{-1-\alpha} g^{\varepsilon} d t d v-\varepsilon \int_{0}^{s} \int_{\Pi_{\tau}} r^{-1-\alpha}\left|f_{x}^{\varepsilon}\right| d x d v d t \\
& \quad+\varepsilon \int_{0}^{s} \int_{\Pi_{\tau}}\left|\frac{\partial r^{-1-\alpha}}{\partial x}\right|\left|f^{\varepsilon}\right| d x d v d t
\end{aligned}
$$


where the constant $C$ depends on the $C^{0,1}$-norms of $\mathbf{E}$ and $B$.

For the other derivatives we get the same kind of estimates:

$$
\begin{aligned}
& \int_{\Pi_{s}}\left|f_{v}^{\varepsilon}\right| d x d v-\int_{\gamma_{0, s}^{+}} \hat{v}_{1}(v)\left|f_{v}^{\varepsilon}\right| d t d v \\
& \quad \leq C+C \int_{0}^{s} \int_{\Pi_{\tau}}\left(\left|f_{x}^{\varepsilon}\right|+\left|\nabla_{v} f^{\varepsilon}\right|\right) d x d v d t+\varepsilon \int_{0}^{s} \int_{\Pi_{\tau}}\left|\nabla_{v} r^{-1-\alpha}\right| f^{\varepsilon} d x d v d t .
\end{aligned}
$$

Therefore two different kinds of terms appear in these inequalities and remain to be estimated:

(i) the first one is

$$
\varepsilon \int_{\gamma_{0, s}^{+}}\left|v_{1}\right|^{-1-\alpha} g_{0}^{\varepsilon} d t d v \leq C \varepsilon^{1-\alpha}\left(\left\|g^{\varepsilon}\right\|_{1}+\epsilon^{\alpha}\left\|g^{\varepsilon}\right\|_{1}\right)
$$

since $g_{0}^{\epsilon}=0$, for $\left|v_{1}\right| \leq \varepsilon$ and $\sup _{v_{1}}\left|g_{0}^{\varepsilon}\left(v_{1}, v_{2}\right)\right| \leq g_{1}\left(v_{2}\right)$, where $g_{1}$ is a function of $v_{2}$

(ii) the second term is

$$
\varepsilon \int_{0}^{s} \int_{\Pi_{\tau}}\left|\nabla_{x, v} r^{-1-\alpha}\right| f^{\varepsilon} d x d v d \tau
$$

On the one hand, we note that

$$
\left|\nabla_{x, v} r^{-1-\alpha}\right| \leq C r^{-2-\alpha}
$$

On the other hand, we previously got an estimate on $f^{\varepsilon}$ :

$$
f^{\varepsilon} \leq M \exp \left(-\varepsilon C r^{-\alpha}\right) \text {. }
$$

Hence, passing to spherical coordinate we prove that

$$
\begin{aligned}
\varepsilon \int_{0}^{s} \int_{\Pi_{\tau}}\left|\nabla_{x, v} r^{-1-\alpha}\right| f^{\varepsilon} d x d v d \tau & \leq C \int_{0}^{\infty} C r^{-1-\alpha} \exp \left(-\varepsilon r^{-\alpha}\right) d r \\
& \leq C \int_{0}^{\infty} \exp (-y) d y \leq C
\end{aligned}
$$

Therefore, it yields

$$
\begin{aligned}
& \int_{\Pi_{s}}\left(\left|f_{x}^{\varepsilon}\right|+\left|f_{v}^{\varepsilon}\right|\right) d x d v-\int_{\gamma_{0, s}^{+}} \hat{v}_{x}(v)\left(\left|f_{x}^{\varepsilon}\right|+\left|f_{v}^{\varepsilon}\right|\right) d v \\
& \leq C+C \int_{0}^{s} \int_{\Pi_{\tau}}\left(\left|f_{x}^{\varepsilon}\right|+\left|f_{v}^{\varepsilon}\right|\right) d x d v d \tau
\end{aligned}
$$

which allows us to conclude from a Gronwall lemma that

$$
\int_{\Pi_{s}}\left(\left|f_{x}^{\varepsilon}\right|+\left|f_{v}^{\varepsilon}\right|\right) d x d v \leq C \exp (C s)
$$

From (3.13) we also deduce that the time derivative of $f^{\varepsilon}$ is bounded in $L^{\infty}\left(0, T, L^{1}(\Omega)\right)$. Finally, $f^{\epsilon}$ is uniformly bounded in $W^{1,1}(\Pi)$.

After the extraction of a subsequence there exists a function $f \in B V \cap L^{\infty}\left(0, T ; L^{1}(\Omega)\right)$ such that

$$
f^{\varepsilon} \rightarrow f, \text { strong in } L_{l o c}^{1}(\Pi), \quad \text { as } \varepsilon \rightarrow 0 .
$$


The traces of $f$ on $\Pi_{0}, \gamma_{0}^{+}$and $\gamma_{1}^{-}$are defined as traces of $B V$ functions, and we have

$$
\left.f\right|_{\Pi_{0}}=f_{0},\left.\quad f\right|_{\gamma_{0}^{+}}=g_{0},\left.\quad f\right|_{\gamma_{1}^{-}}=0 .
$$

Moreover,

$$
\varepsilon r^{-1-\alpha} f^{\varepsilon} \rightarrow 0, \quad \text { as } \varepsilon \rightarrow 0,
$$

and we deduce that $f$ is a solution to the linear Vlasov equation (1.1). The uniqueness among $B V$ solutions is obvious since the problem is linear, and their trace is well-defined and given.

4. Proof of Theorem 1.1. In this section, we construct an iteration scheme to prove the existence of a solution to (1.1) coupled with (1.2)-(1.4). For each $n \geq 0$, we define a sequence of approximate solutions $f^{n}(t, x, v), \mathbf{E}^{n}(t, x)$ and $B^{n}(t, x)$. We begin with

$$
f^{0}(t, x, v)=f_{0}(x, v), \quad \mathbf{E}^{0}(t, x)=\mathbf{E}_{0}(x), \quad B^{0}(t, x)=B_{0}(x) .
$$

We define $f^{n}$ iteratively by

$$
\frac{\partial f^{n}}{\partial t}+\hat{v}_{x}(v) \frac{\partial f^{n}}{\partial x}+\left(\mathbf{E}^{n-1}+\hat{v}(v) \times \mathbf{B}^{n-1}\right) \cdot \nabla_{v} f^{n}=0, \quad x \in(0,1), v \in \mathbb{R}^{2},
$$

with initial datum

$$
f^{n}(x, v)=f_{0}(x, v), \quad x \in(0,1), \quad v \in \mathbb{R}^{2} .
$$

From $f^{n}$, we can define

$$
\rho^{n}(t, x)=\int_{\mathbb{R}^{2}} f^{n}(t, x, v) d v, \quad j^{n}(t, x)=\int_{\mathbb{R}^{2}} \hat{v}(v) f^{n}(t, x, v) d v,
$$

and then $\mathbf{E}^{n}$ and $B^{n}$ are taken to be the solutions to

$$
\begin{aligned}
\frac{\partial E_{1}^{n}}{\partial t} & =-j_{x}^{n}(t, x), \quad t \in \mathbb{R}^{+}, x \in(0,1), \\
\frac{\partial E_{2}^{n}}{\partial t}+\frac{\partial B^{n}}{\partial x} & =-j_{y}^{n}(t, x), \quad t \in \mathbb{R}^{+}, x \in(0,1), \\
\frac{\partial B^{n}}{\partial t}+\frac{\partial E_{2}^{n}}{\partial x} & =0, \quad t \in \mathbb{R}^{+}, x \in(0,1) .
\end{aligned}
$$

Applying Lemma 2.2 we first establish uniform estimates on the $L^{\infty}$ norms on the electromagnetic fields $\mathbf{E}^{n}$ and $B^{n}$. These bounds only depend on the energy identity (2.2),

$$
\left\|\mathbf{E}^{n}\right\|_{\infty}+\left\|B^{n}\right\|_{\infty} \leq C .
$$

From this bound, we ensure the control of the support to the solution $f^{n}$,

$$
P^{n}(t)=\sup \left\{|v|: f^{n}(t, x, v) \neq 0, \quad(t, x) \in[0, T] \times[0,1]\right\},
$$

which satisfies

$$
P^{n}(t) \leq R_{0}+\int_{0}^{t}\left(\left\|\mathbf{E}^{n}(s)\right\|_{\infty}+\left\|B^{n}(s)\right\|_{\infty}\right) d s \leq C,
$$

where $R_{0}$ is a bound on the support of the initial datum.

Then, as we show above in Lemma 2.3, the fields are uniformly bounded in $C^{0,1}$ :

$$
\left\|\mathbf{E}^{n}\right\|_{C^{0,1}}+\left\|B^{n}\right\|_{C^{0,1}} \leq C .
$$


Finally, we apply Proposition 3.4 to get a uniform $B V$ bound on the distribution function $f^{n}$.

After the extraction of a subsequence there exists a function $f \in B V \cap L^{\infty}\left(0, T ; L^{1}(\Omega)\right)$ such that

$$
f^{n} \rightarrow f, \text { strong in } L_{l o c}^{1}(\Pi), \quad \text { as } n \rightarrow \infty
$$

and

$$
\left(E^{n}, B^{n}\right) \rightarrow(E, B), \text { strong in } L^{\infty}(\Pi), \quad \text { as } n \rightarrow \infty .
$$

To conclude the existence proof, we proceed as in $[8$ and show directly that $f, E$ and $B$ are solution to (1.1) and (1.2)-(1.4).

To prove uniqueness, we consider two solutions $\left(f_{1}, \mathbf{E}_{1}, B_{1}\right)$ and $\left(f_{2}, \mathbf{E}_{2}, B_{2}\right)$ in the class of solutions we constructed. We set

$$
\tilde{f}=f_{1}-f_{2}, \tilde{E}=\mathbf{E}_{1}-\mathbf{E}_{2}, \tilde{B}=B_{1}-B_{2},
$$

which are solutions to

$$
\frac{\partial \tilde{f}}{\partial t}+\hat{v}_{1}(v) \frac{\partial \tilde{f}}{\partial x}+\left(\mathbf{E}_{1}+\hat{v}(v) \times \mathbf{B}_{1}\right) \cdot \nabla_{v} \tilde{f}+(\tilde{\mathbf{E}}+\hat{v}(v) \times \tilde{\mathbf{B}}) \cdot \nabla_{v} f_{2}=0,
$$

and for the difference of the electromagnetic field,

$$
\begin{aligned}
\frac{\partial \tilde{E}_{1}}{\partial t} & =-\tilde{j}_{1}(t, x), \\
\frac{\partial \tilde{E}_{2}}{\partial t}+\frac{\partial \tilde{B}}{\partial x} & =-\tilde{j}_{2}(t, x), \\
\frac{\partial \tilde{B}}{\partial t}+\frac{\partial \tilde{E}_{2}}{\partial x} & =0,
\end{aligned}
$$

where $\tilde{\rho}=\tilde{\rho}_{1}-\tilde{\rho}_{2}, \tilde{j}=\tilde{j}_{1}-\tilde{j}_{2}$. On the one hand, from (4.4) we obtain

$$
\frac{d}{d t}\|\tilde{f}(t)\|_{1} \leq\left(\|\tilde{E}(t)\|_{\infty}+\|\tilde{B}(t)\|_{\infty}\right) T V\left(f_{2}\right) .
$$

On the other hand, we proceed as in Lemma 2.2 we multiply (4.4) by $\operatorname{sgn}(f) \sqrt{1+v^{2}}$ and integrate by parts,

$$
\frac{\partial e}{\partial t}-\frac{\partial m}{\partial x}=-\tilde{j} \tilde{E}+E_{1} \int_{\mathbb{R}^{2}} \hat{v}|\tilde{f}| d v+\tilde{E} \int_{\mathbb{R}^{2}} \hat{v} \operatorname{sgn}(\tilde{f}) f_{2} d v,
$$

with

$$
\begin{aligned}
e & =\frac{1}{2}\left(|\tilde{\mathbf{E}}|^{2}+|\tilde{B}|^{2}\right)+\int_{\mathbb{R}^{2}} \sqrt{1+|v|^{2}}|\tilde{f}| d v, \\
m & =-\int_{\mathbb{R}^{2}} v_{1}|\tilde{f}| d v-E_{2} B .
\end{aligned}
$$

Thus, we get the following estimate for $0 \leq t_{0}, t_{1} \leq t$ :

$$
\begin{array}{r}
\int_{t_{0}}^{t}(e+m)(\tau, x-t+\tau) d \tau+\int_{t_{1}}^{t}(e+m)(\tau, x-t+\tau) d \tau \\
\leq C+C \int_{0}^{t}\left(\|\tilde{\mathbf{E}}(\tau)\|_{\infty}+\|\tilde{B}(\tau)\|_{\infty}+\|\tilde{f}(\tau)\|_{1}\right) d \tau .
\end{array}
$$




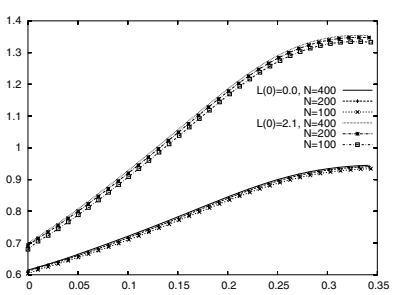

(1)

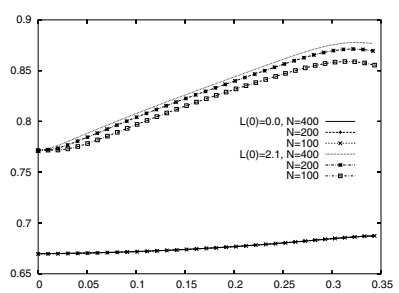

(2)

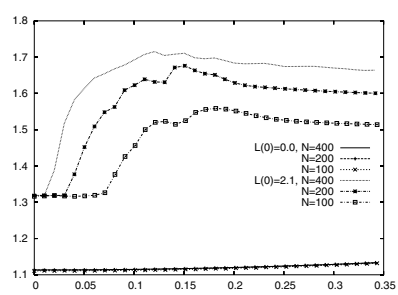

(3)

Fig. 2. Time evolution of (1) the $B V$-norm, (2) $L^{4}$-norm and (3) $L^{\infty}$ norm of $\nabla f$ for different mesh sizes

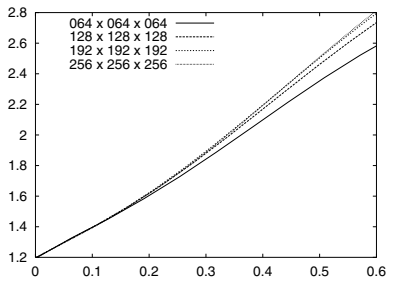

(1)

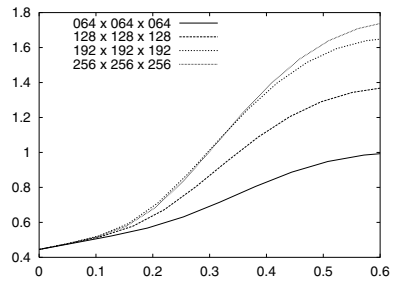

(2)

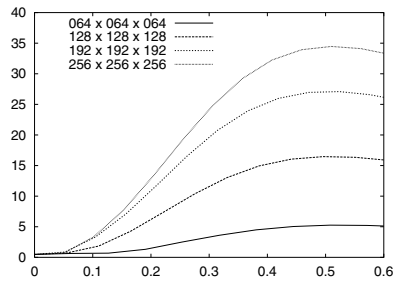

(3)

FIG. 3. Time evolution of (1) the $B V$-norm, (2) $L^{2}$ norm of $\nabla f$ and

(3) $L^{\infty}$ norm of $\nabla f$ for different mesh sizes

Finally, using

$$
\int_{0}^{t} \frac{\left|v_{2}\right|}{\sqrt{1+|v|^{2}}}|\tilde{f}|(\tau, x \pm t \mp \tau) d v d \tau \leq \int_{0}^{t}(e \pm m)(\tau, x \pm t \mp \tau) d \tau
$$

we obtain

$$
\begin{aligned}
\|\tilde{\mathbf{E}}(t)\|_{\infty}+\|\tilde{B}(t)\|_{\infty} & \leq C_{0}+\left\|k^{+}(t)\right\|_{\infty}+\left\|k^{-}(t)\right\|_{\infty} \\
& \leq C+C \int_{0}^{t}\left(\|\tilde{\mathbf{E}}(\tau)\|_{\infty}+\|\tilde{B}(\tau)\|_{\infty}+\|\tilde{f}(\tau)\|_{1}\right) d \tau .
\end{aligned}
$$

The uniqueness result follows by gathering (4.8) and (4.9), and applying the Gronwall lemma.

5. Numerical simulations. In this section, we present some numerical simulations obtained with two different methods: an up-wind WENO method [15, 20] and a semiLagrangian method proposed in [7. Of course a complete mathematical analysis for such schemes is very difficult, but in some simplified cases it is possible to prove that the numerical solution converges to the solution of the continuous problem even when the solution is only $B V$ (see for instance [6] for the Vlasov-Poisson system with boundary conditions). Here, we present numerical results illustrating the singularity formation for the Vlasov-Maxwell system of one spatial dimension and one or two phase space dimensions. 

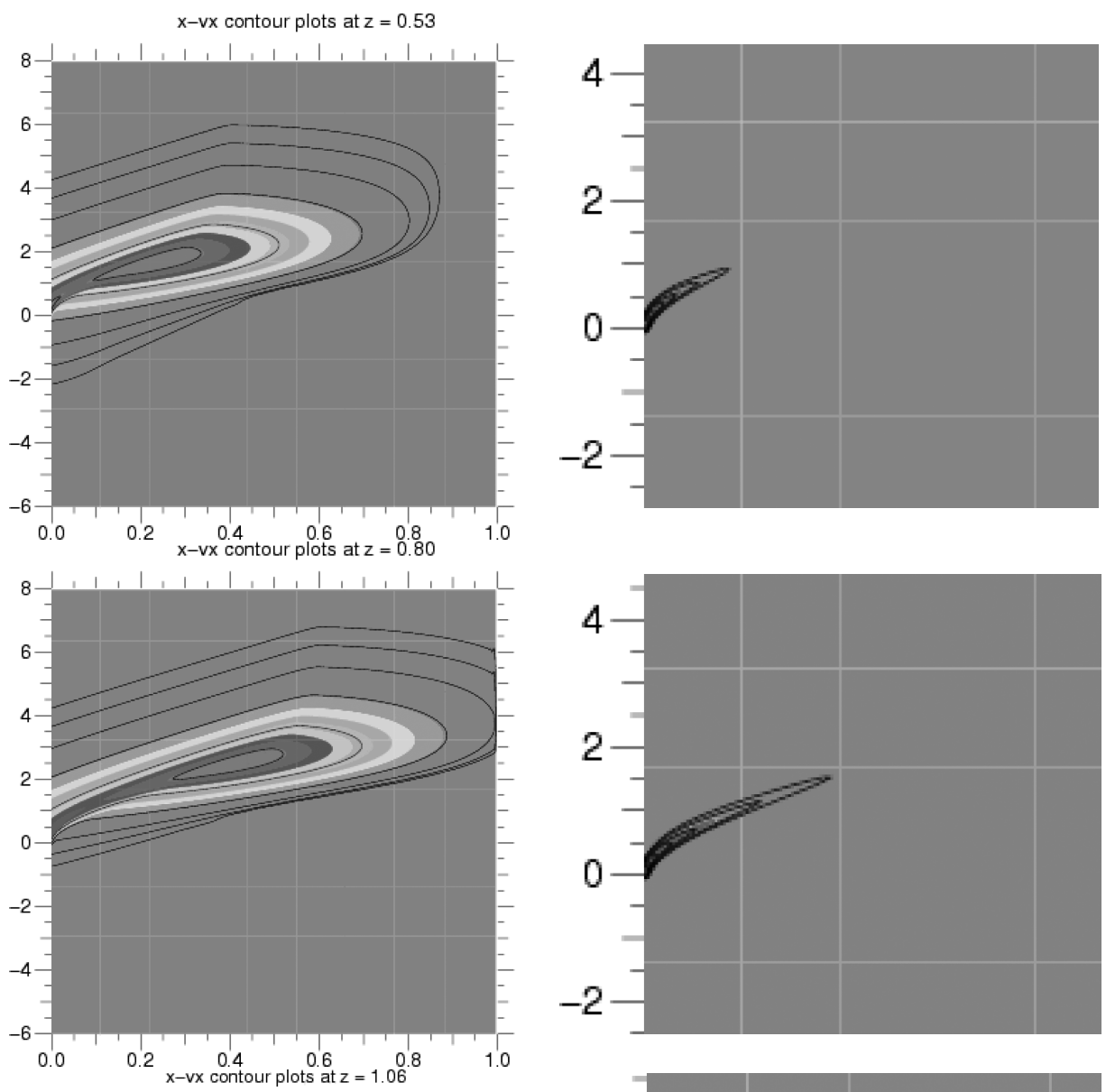

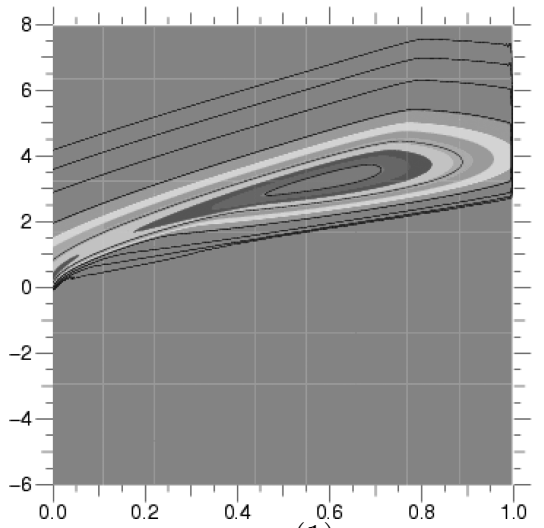

(1)

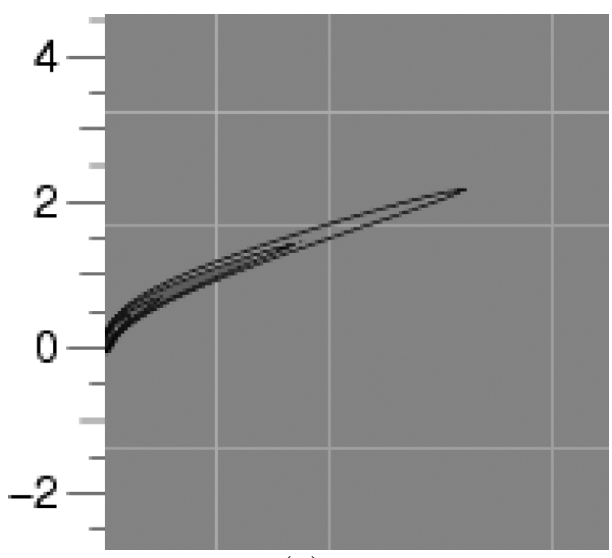

$(2)$

FIG. 4. Left: time development of the $\left(x, v_{x}\right)$ projection of the distribution function at time $t=0.25$ (top); 0.45 (middle) and 0.95 (bottom). Right: time development of the $\left(x, v_{x}\right)$ projection of the gradient of the distribution function with respect to $x$ at time $t=0.25$ (top); 0.45 (middle) and 0.95 (bottom) 
5.1. The Vlasov-Poisson system. In [12, the authors proved that the one-dimensional Vlasov-Poisson boundary value problem admits a unique $B V$ solution. Moreover, setting

$$
\lambda(t)=\int_{0}^{1} E(t, x) d x>0,
$$

they have shown that the solution $f$ is discontinuous when

$$
\lambda(0)>\int_{0}^{1} \int_{\mathbb{R}}(1-x) f_{0}(x, v) d v d x .
$$

We consider the boundary value problem

$$
\frac{\partial f}{\partial t}+v \frac{\partial f}{\partial x}+E \frac{\partial f}{\partial v}=0, \quad t \in \mathbb{R}^{+}, x \in(0,1), v \in \mathbb{R},
$$

coupled with the Poisson equation

$$
\frac{\partial E}{\partial x}=\rho=\int_{\mathbb{R}} f(t, x, v) d v, \quad \int_{0}^{1} E(t, x) d x=\lambda_{0}
$$

and

$$
f_{0}(x, v)=n_{0}(x) \frac{1}{\sqrt{2 \pi}} v^{2} \exp \left(-v^{2} / 2\right),
$$

where

$$
n_{0}(x)= \begin{cases}(1+\gamma x)\left(1-4 x^{2}\right)^{4}, & x \in(0,1 / 2) \\ 0, & \text { else }\end{cases}
$$

and

$$
g(t, v)=\frac{1}{\sqrt{2 \pi}} v^{2} \exp \left(-v^{2} / 2\right) .
$$

We performed several simulations with $n_{x}=n_{v}=N$ and $N=100,200$ and 400 points per direction for different values of $\lambda_{0}$. Thus, we computed the discrete $W^{1, p}$ norms

$$
\|f\|_{N, p}^{p}=\Delta v \sum_{i, j=1}^{N}\left|f_{i, j}-f_{i-1, j}\right|^{p}+\Delta x \sum_{i, j=1}^{N}\left|f_{i, j}-f_{i, j-1}\right|^{p}, \quad p>1 .
$$

On the one hand, we performed a simulation when the condition (5.1) is well satisfied; then we expect to capture the discontinuous solution. In this case, we know that the total variation of the solution is bounded, whereas the $L^{p}$ norm of the gradient is not finite for $p>1$. On the other hand, when the condition (5.1) is not satisfied, we do not know if the solution is continuous or not. Thus, we plot in Fig. 2 the evolution of the discrete $W^{1, p}$ norm for $p=1,4$ and $\infty$. These numerical results perfectly illustrate that for these two values of $\lambda(0)=0$ and $\lambda(0)=2.10947$, for which the condition (5.1) is satisfied, the behavior of the solution is different. While for the former case with $\lambda(0)=0$ there is convergence with mesh refinements for all norms, for the latter case $\lambda(0)=2.10947$ it seems that there is such convergence with mesh refinements only for the BV norm. We have also performed numerical simulations for intermediate values of $\lambda(0)$, when (5.1) is not satisfied, and observed that in this situation the numerical solution is smooth, but it is difficult to approximate accurately the threshold. 
5.2. The Vlasov-Maxwell system. We consider the system (1.1)-1.4 with a zero initial condition $f(0, x, v)=0$ and $\mathbf{E}(0)=0, B(0)=1$ and boundary conditions

$$
\begin{aligned}
& f(t, 0, v)=g(t, v)=\frac{1}{2 \pi}|v|^{2} \exp \left(-\frac{|v|^{2}}{2}\right) \frac{t^{2}}{1+t^{2}}, v_{x}>0, \\
& f(t, 1, v)=0, v_{x}<0 .
\end{aligned}
$$

We performed several simulations with $n_{x}=n_{v_{x}}=n_{v_{y}}=N$ and $N=100,150$ and 200 points per direction.

In Fig. 3. we first report the evolution of the total variation of $f_{N}$ :

$$
T V\left(f_{N}\right)=\sum_{i, j, k=1}^{N} \Delta v_{x} \Delta v_{y}\left|f_{i+1, j, k}-f_{i, j, k}\right|,
$$

where $f_{i, j, k}$ represents an approximation of the distribution function $f\left(x_{i}, v_{x j}, v_{y k}\right)$ on the phase space grid. We also present the evolution of the approximation to the $L^{\infty}$ norm of $\nabla f$. We first observe that the evolution of the $B V$ norm of $f_{N}$ is not sensitive to the mesh size, which means that it is described correctly. On the other hand, the time evolution of the discrete $L^{\infty}$ norm of $\nabla f_{N}$ strongly depends on the number of mesh points. Moreover, $\left|\nabla f_{N}\right|_{\infty}$ goes to infinity when the number of points is increasing, which means that the solution of the continuous Vlasov-Maxwell system does not belong to $W^{1, \infty}$ as we showed before.

The use of the WENO method allows us to describe accurately the evolution of the solution without introducing numerical oscillations even if the solution is not smooth. Thus, we represent the contour plots of the projection of the distribution function and its gradients in the phase space $x-v_{1}$ in Fig. 4. We observe that the singularity arises from the point $\left(x, v_{1}\right)=(0,0)$ and is propagating in time into the domain.

\section{REFERENCES}

[1] Arsen'ev, A.A., Global existence of a weak solution of Vlasov's system of equations, Zh. vychisl. Mat. i. Mat. Fiz. 15 (1975), 136-147. MR0371322 (51:7541)

[2] Asano, K., On local solutions of the initial value problem for the Vlasov-Maxwell equation, Comm. Math. Phys. 106 (1986), 551-568. MR0860309 (87k:76071)

[3] Bardos, C. and Degond, P., Global existence for the Vlasov-Poisson equation in 3 space variables with small initial data, Ann. Inst. H. Poincaré Anal. Non Linéaire 2 (1985), 101-118. MR0794002 (86k:35129)

[4] Degond, P. and Raviart, P.-A., An asymptotic analysis of the Vlasov-Poisson system: The Childlangmuir law, Asymptotic Anal. 4 (1991), no. 3, 187-214. MR.1115929 (92f:82062)

[5] DiPerna, R.J. and Lions, P.-L., Global existence of weak solution for the Vlasov-Maxwell system, Comm. Pure Appl. Math. 42 (1989), 729-757. MR1003433 (90i:35236)

[6] Filbet, F., Convergence of a finite volume scheme for the Vlasov-Poisson system, SIAM J. Numer. Anal. 39 (2001), 1146-1169. MR1870837 (2002i:65089)

[7] Filbet, F., Sonnendrücker, E. and Bertrand, P., Conservative numerical schemes for the Vlasov equation, J. Comput. Phys. 172 (2001), 166-187. MR1852326 (2002h:76106)

[8] Glassey, R. and Schaeffer J., On the one and one-half dimensional relativistic Vlasov-Maxwell system, Math. Methods Applied Sciences, 13, (1990) 169-179. MR1066384 (91g:82054)

[9] Glassey, R. and Schaeffer, J., The "two and one-half dimensional" relativistic Vlasov Maxwell system, Comm. Math. Phys. 185 (1997), 257-284. MR1463042 (98f:35143)

[10] Glassey, R. and Strauss, W., Large velocities in the relativistic Vlasov-Maxwell equations, J. Fac. Sci. Univ. Tokyo Sect. IA Math. 36 (1989), 615-627. MR1039487(91b:82059) 
[11] Guo, Y., Singular solutions of the Vlasov-Maxwell system on a half line, Arch. Rational Mech. Anal. 131 (1995), 241-304. MR.1354697 (96h:35228)

[12] Guo, Y., Shu, C.-W. and T. Zhou, The dynamic of a plane diode, SIAM J. Math. Anal. 35 (2004), 1617-1635. MR2083792 (2005f:35302)

[13] Greengard, C. and Raviart, P-A., A boundary-value problem for the stationary Vlasov-Poisson equation: the plane diode, Comm. Pure. Appl. Math. 43 (1990), 472-507. MR1047333 (91b:82064)

[14] Horst, E., On the classical solutions of the initial value problem for the unmodified non-linear Vlasov equation, Math. Methods Appl. Sci. 3 (1981), 229-248. MR0657294 (83h:35110)

[15] Jiang, G. and Shu, C.-W., Efficient implementation of weighted ENO schemes, J. Comput. Phys. 126 (1996), 202-228. MR 1391627 (97e:65081)

[16] Lions, P.-L. and Perthame, B., Propagation of moments and regularity for the 3-dimensional VlasovPoisson system, Invent. Math. 105 (1991), 415-430. MR.1115549 (92e:35160)

[17] Pfaffelmoser, K., Global classical solutions of the Vlasov-Poisson system in three dimensions for general initial data, J. Differential Equations 95 (1992), 281-303. MR.1165424 (93d:35170)

[18] Poupaud, F., Boundary value problems for the stationary Vlasov-Maxwell system, Forum Math., 4 (1992), no. 5, 499-527. MR.1176884 (93i:35144)

[19] Schaeffer, J., Global existence of smooth solutions to the Vlasov-Poisson system in three dimensions, Comm. Partial Differential Equations 16 (1991), 1313-1335. MR.1132787 (92g:82113)

[20] Shu, C.-W., Essentially non-oscillatory and weighted essentially non-oscillatory schemes for hyperbolic conservation laws, Advanced Numerical Approximation of Nonlinear Hyperbolic Equations, B. Cockburn, C. Johnson, C.-W. Shu and E. Tadmor (Editor: A. Quarteroni), Lecture Notes in Mathematics, Springer 1697 (1998), 325-432. MR.1728856(2001a:65096) 